\title{
Plasma vasopressin and response to treatment in primary nocturnal enuresis
}

\author{
H Devitt, P Holland, R Butler, E Redfern, E Hiley, G Roberts
}

\begin{abstract}
Aims-To examine the relation between nocturnal vasopressin release and response to treatment with the vasopressin analogue 1-desamino-8-D-arginine vasopressin (DDAVP) in children with primary monosymptomatic nocturnal enuresis.

Design-Children were recruited from a specific enuresis clinic and entered into a defined treatment programme. Nocturnal vasopressin concentrations were measured every 15 minutes over a four hour period during overnight admission. Results-Sixty seven children were eligible for entry into the study, 35 of whom agreed to overnight sampling. There was a quadratic relation between mean plasma AVP and response to treatment with DDAVP, with very high or very low concentrations being unresponsive. Plasma AVP profiles ranged from low concentrations with little variability to high concentrations with wide variability. Conclusion-The ability to respond to DDAVP is related to endogenous AVP production and is influenced by neuronal patterning in early infancy. The best predictors of success with treatment were a past history of breast feeding, mean nocturnal AVP concentration, and the height of the child. The response was adversely affected by poor weight at birth and poor linear growth. The study suggests differing causes of nocturnal enuresis related to different patterns of AVP release.
\end{abstract}

(Arch Dis Child 1999;80:448-451)

Paediatrics General

Infirmary, Leeds

LS2 9NS, UK

H Devitt

P Holland

Clinical Psychology,

Leeds Community and

Mental Health NHS

Trust, Leeds LS2 9HM,

UK

R Butler,

E Hiley

G Roberts

Department of

Statistics, University of

Leeds, Leeds LS2 9JT,

UK

E Redfern

Correspondence to:

Dr Holland.

Accepted 13 January 1999 medication, rather than the cure rate off medication. In a review of 18 randomised trials, Moffat and colleagues found that, although in all the studies the wetting frequency was reduced on DDAVP, only $25 \%$ of children became dry on DDAVP when dryness was defined as 14 consecutive dry nights. ${ }^{4}$ It is not clear why only a proportion of children respond if a low concentration of AVP is the physiological reason for enuresis.

Our study investigated this issue by examining both the relation between nocturnal AVP plasma concentrations and response to DDAVP, together with an analysis of which physiological factors might influence the response.

\section{Methods}

Children were recruited to a special nocturnal enuresis clinic established in the paediatric department of Leeds General Infirmary. The clinic was run weekly and staffed by a research paediatrician and assistant clinical psychologist. Children 8 years or over with nocturnal enuresis were initially evaluated in the clinic and if they fulfilled the criteria (table 1) they entered a standard treatment regimen. Those who failed to fulfil the criteria were followed up in a separate general paediatric clinic and were excluded from our study.

All children were weighed to $0.1 \mathrm{~kg}$ using electronic scales and measured to $0.1 \mathrm{~cm}$ using a standard stadiometer at their first clinic appointment. A detailed history was taken of the early perinatal period, noting in particular whether the child was breast fed or bottle fed, and for what length of time. Children were classified as breast fed for $>3$ months or $>6$ months if this was the principal method of feeding for the child during these periods, allowing for the occasional bottle feed. A retrospective history of maternal smoking in pregnancy was recorded. Parents were asked whether any first or second degree relatives were known to have nocturnal enuresis, defined as still wet at 6 years or above. Social class was defined as dependent on parental profession.

Children were started on a treatment programme using either DDAVP spray $20 \mu \mathrm{g}$ at night (Desmospray; Ferring, Langley, Berks) or DDAVP tablets $0.2 \mathrm{mg}$ at night (Desmotabs; Ferring), according to individual choice. Clear instructions were given on the method of

Table 1 Criteria for study entry

Aged 8-16 years

Wet at least four nights a week

No associated daytime wetting

Has never been dry $>6$ months

No know neurological or urological abnormality

Mainstream education without statement

Informed parental and child consent 


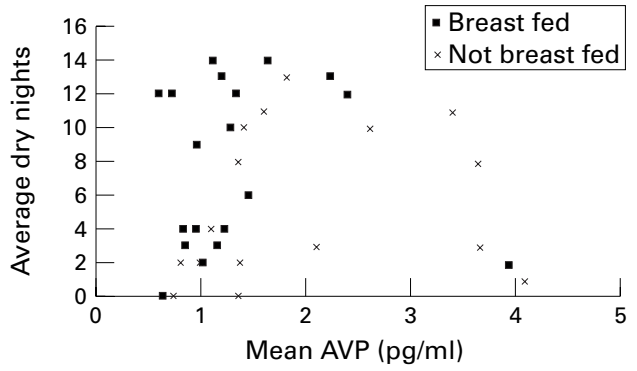

Figure 1 Mean nocturnal AVP concentration in relation to the average number of dry nights on treatment with $D D A V P$. Children are divided into those who were breast fed and not breast fed in the first 3 months after birth.

administration and potential side effects. The dose was doubled after two weeks if there was failure to achieve continence. Success was defined as achieving 10 or more dry nights out of 14, maintained over a four week period while on DDAVP. Those failing to respond to treatment continued through a structured treatment programme and are not reported in this paper.

After informed consent those children who agreed were admitted overnight. Blood samples were taken every 15 minutes, via an indwelling cannula, from 23:00 to 03:00. Blood was separated and frozen at $-20^{\circ} \mathrm{C}$ within 20 minutes of sampling. Blood was subsequently analysed in the department of chemical pathology, Royal Gwent Hospital, Newport for AVP using an established technique involving extraction and radioimmunoassay. Concentrations were expressed in $\mathrm{pg} / \mathrm{ml} .^{5}$ The fluid intake for each child was standardised from 18:00 on the evening of admission, each child being allowed one beaker of water $(300 \mathrm{ml})$ at bedtime. Because we wanted to study the children under as near normal circumstances as possible, they were allowed to follow their normal drinking patterns during the day.

Ethics permission for the study was granted by the United Leeds Teaching Hospital ethics committee.

DATA ANALYSIS

Data were examined independently at the department of statistics, University of Leeds by one author (ER). To identify factors influencing successful treatment, a general linear regression model analysis was carried out using

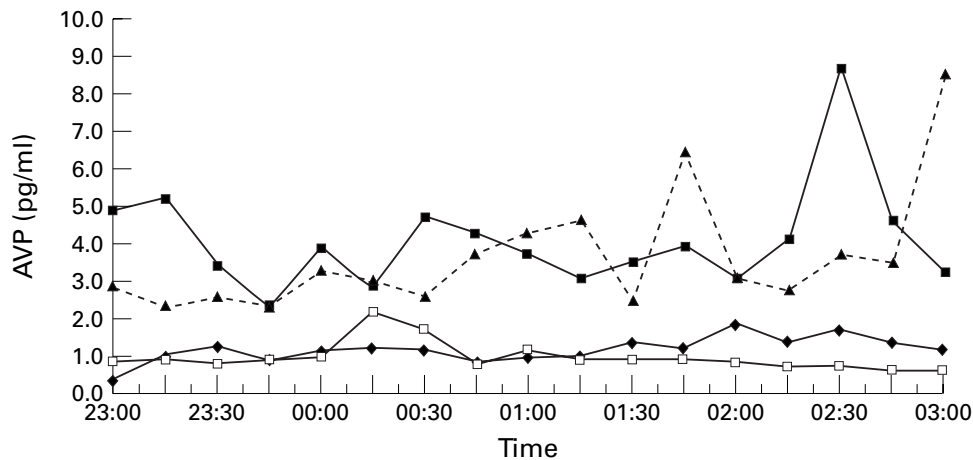

Figure 2 Two patterns of AVP release, one with low mean values and little variability and one with higher means and wide variability. both classification variables and a set of possible covariants. Product moment and Spearman rank correlation coefficients were used to identify possible relations between variables, the choice of coefficients depending on the type of variables involved. The effect of class variables, such as breast feeding and smoking, on both success and AVP concentrations was analysed using the Welch-Aspin modification of the Student's $t$ test (allowing for unequal variance) and the Mann-Whitney independent sample test.

Statistical analysis of AVP was based both on the average of the plasma values over the four hour collection period and on the variability of the plasma concentrations. The variance of the plasma AVP value from the mean was calculated, with a variable pattern being defined as at least one peak of AVP greater than three times the coefficient of variation for the assay.

\section{Results}

One hundred and twenty one children were referred to the clinic between July 1994 and May 1996, 67 of these children (55 boys, 12 girls) met the criteria for entry into our study, with an age range from 8 to 14.5 years. Of those eligible for entry 35 (28 boys, seven girls) agreed to overnight blood sampling (mean age, 10.5 years; range, $8-14)$.

Of the 35 children, 14 met the criterion of success with DDAVP treatment. The mean plasma AVP concentrations ranged from $0.59 \mathrm{pg} / \mathrm{ml}$ to $4.09 \mathrm{pg} / \mathrm{ml}$. There was a significant relation between the mean AVP (pg/ $\mathrm{ml})$ and response to treatment $(\mathrm{p}<0.001)$. There was evidence of a quadratic response against the mean with very low concentrations and very high concentrations being unresponsive to treatment (fig 1).

Two distinct patterns of AVP release were observed: non-variable and variable. In 17 of 35 children there was little variability over the collecting period, with a mean (range) plasma AVP of $0.99 \mathrm{pg} / \mathrm{ml}(0.59-1.36)$. A second group (14 of 35 ) had significantly higher AVP concentrations with wide variability ( $\mathrm{p}<0.0001$ ), and a mean (range) plasma AVP of $2.48 \mathrm{pg} / \mathrm{ml}$ (1.09-4.09). The variability was not associated with either waking or changing posture. Examples of two children in each group are shown in fig 2 . Four children could not be clearly assigned to either group because of insufficient data points. About one third of the non-variable group responded to DDAVP compared with over half of the variable group. This was not significant (Fisher's exact test, $\mathrm{p}=0.36$ ).

Within the cohort of 35 children, 18 had previously been breast fed for $>3$ months but no child had been breast fed for $>6$ months. Breast fed children had significantly lower concentrations of AVP (confidence limits, 0.032 to $1.153 ; p=0.036$ ) (fig 1) and showed less variance $(\mathrm{p}=0.027$ ) (fig 3 ). Breast fed children were significantly more likely to respond to treatment with DDAVP.

The height of the child at the time of entry into our study was shown to influence response to DDAVP independent of breast feeding. 


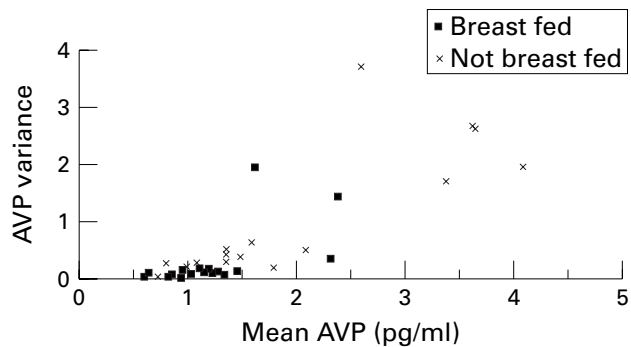

Figure 3 Influence of breast feeding on variability of AVP release, showing low variability in children previously breast fed.

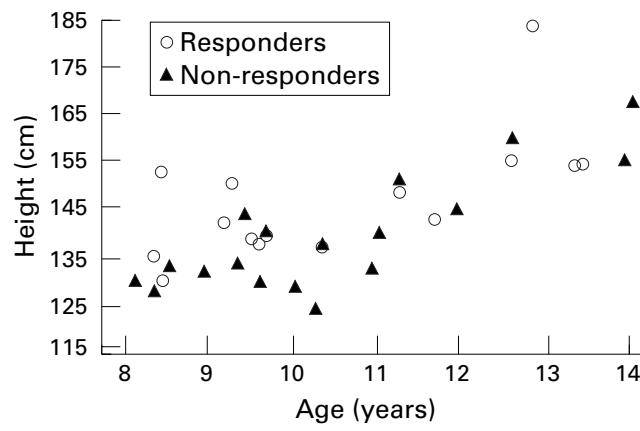

Figure 4 Effect of both age and height on responsiveness to DDAVP treatment, with short young children being the least responsive.

Children with poor linear growth were less likely to respond to treatment $(p<0.01)$, the least likely being the youngest and shortest (fig 4).

A generalised linear model analysis of the number of dry nights was undertaken using AVP group (variable or non-variable), breast feeding, and height as covariants. This suggested that there were significant effects for breast feeding $(p=0.023)$ and height $(\mathrm{p}=0.016)$ but not for the AVP group $(p=0.071)$. The best predictors of success were breast feeding, mean AVP and its square, and the height of the child.

A history of enuresis in a first degree relative was found in 27 of 35 children. AVP concentrations and variance or response to DDAVP was found to have no association with a family history of enuresis involving first or second degree relatives.

\section{Discussion}

The attainment of nocturnal continence appears in part to depend on the development of a circadian rise in AVP resulting in reduced urine production at night. Neuronal networks involving the hypothalamic pituitary axis and AVP begin to become established early after birth, ${ }^{6}$ with circadian rhythms being determined from the suprachiasmatic nucleus (SCN), one of the three hypothalamic nuclei associated with AVP production. A number of studies have provided evidence for this circadian rise, although a previous study has shown wide variation in AVP concentrations, suggesting that frequent sampling is required to obtain an accurate picture of plasma AVP release at night. ${ }^{7}$

In children with low concentrations of AVP there is a direct relation between the mean plasma AVP concentration and response (fig 1). The lower the AVP the less likely the child is to respond to DDAVP. The confounding factor is the influence of previous breast feeding on response to DDAVP. Breast feeding is known to enhance early neuronal maturation and visual acuity, which may be important in the entrainment of normal circadian rhythmicity and in the establishment of normal neuronal pathways. ${ }^{8}$ One other study has also shown that breast feeding enhances the development of nocturnal continence, making it less likely that this is a chance finding. ${ }^{9}$ It is proposed that children with enuresis and low AVP concentrations, particularly those breast fed as infants, have established their normal neuronal pathways, but there is a failure to produce the normal circadian release of AVP at night. However, this may be mimicked by giving exogenous AVP.

The pattern of AVP release changes with an increasing variability associated with higher plasma concentrations. This may reflect increasing central release as part of establishing the normal circadian rhythm or, as we postulate, it could be related to bladder filling and AVP release stimulated by bladder contraction. Watanabe has examined the relation between sleep state and nocturnal arousal caused by a full bladder and describes, within a subgroup, uninhibited forceful bladder contractions leading to urination without arousal. ${ }^{10}$ Other studies have suggested that bladder distension might result in AVP release. ${ }^{11}$ Therefore, the bladder may try to regulate urine production by initiating AVP release as it fills. Although during the day this eventually results in the desire to micturate, at night, it is necessary to be aroused from sleep first before the bladder empties. Only by specific bladder pressure studies would it be possible to show that high pressure bladder contractions during sleep would result in pulses of AVP as seen in these children.

The attainment of nocturnal continence may first be associated with the development of the circadian rhythm of AVP and then "fine tuned" by the bladder stimulating AVP release once a certain volume is reached. The combination of DDAVP and anticholinergic medication may be useful in patients with nocturnal enuresis and has already been used and shown to be beneficial. $^{12}$

Very high concentrations of plasma AVP (mean values, 3.4-4.1 pg/ml) were seen in five children (fig 1) and were associated with persistent nocturnal enuresis. In this group, there was a wide variance, with peaks of AVP reaching 7-16 pg/ml. Further exogenous AVP is unlikely to help and the aetiology of the nocturnal enuresis might be different in these children from those shown on the rest of the graph. All five children were boys, had severe enuresis, were wet every night, and never woke up to pass urine. Four of five had a known family history of enuresis and all were $>50$ th centile for height. Failure to respond to the action of a hormone (AVP) in association with high concentrations of that hormone raises the possibility of a receptor defect. Genetic linkage 
of nocturnal enuresis to $12 \mathrm{q}$ has led to speculation that the aquaporin 2 gene also found on $12 \mathrm{q}$ might be involved. ${ }^{13}$ This has been questioned because of the responsiveness of nocturnal enuresis to DDAVP, which tends to rule out a receptor defect. It is possible that in these five children there is a failure of a longer acting overnight receptor to respond to high AVP concentrations. ${ }^{14}$ Assuming these five children have a different aetiology of their enuresis, removing them from the previous analysis improves the response to DDAVP in the variable group from 8 of 14 to 8 of 9 , compared with 6 of 17 in the low variability group. This is compatible with the maturation theory, where there is a need for a circadian rise of AVP to occur before continence can be achieved.

Within the whole cohort of 67 children poor linear growth and low birth weight adversely affected the response to DDAVP. ${ }^{15}$ However, there appears to be evidence of maturation, with the older children being more likely to respond (fig 4). Jarvelin et al had previously shown that children with poor linear growth were more likely to have severe enuresis. ${ }^{16}$ Previous studies have shown that the in utero environment is important in hypothalamicpituitary development and that it may be adversely affected by chronic hypoxia. ${ }^{17}$ It is possible that in these children the in utero environment has led to reduced neuronal development of the hypothalamic pituitary axis such that both growth hormone and AVP are produced suboptimally at night.

Our study was designed to investigate specifically children with primary monosymptomatic enuresis. The inclusion criteria were strict to reduce confounding variables to a minimum, so that children with daytime wetting and children with developmental problems were not included. The study confirms the importance of AVP release at night in the establishment of nocturnal continence. In particular we have shown the importance of the hypothalamic axis and how this may be influenced adversely by perinatal events and advantageously by breast feeding. There appear to be at least two possible aetiologies of nocturnal enuresis. One is associated with a low concentration of AVP, where there may be a failure to produce the circadian rise in AVP but normal neural pathways exist. We have suggested that the bladder itself may stimulate AVP release in an attempt to finely tune the control of night time urine production. The second is associated with high AVP concentrations and severe nocturnal enuresis. The aetiology must be speculative but our data suggest that AVP receptor failure might be involved in night time continence.

Jarvelin et al and Fergusson et al have suggested that primary and secondary enuresis are aspects of the same problem, the primary form being regarded as a delay in maturation of the physiological mechanisms that lead to continence. ${ }^{18} 19$ There might be a progressive developmental process in the establishment of nocturnal continence. This involves the development of a circadian rise in AVP resulting in reduction in urine volume. In conjunction with this is the production of AVP in relation to bladder contraction and the ability to awake to a full bladder. Although functional bladder volume might be a contributory factor, it may not be part of the developmental process involved primarily in nocturnal continence. The latter, however, is conditional on daytime continence, which is dependent on the ability to maintain a full bladder and on the development of voluntary control over micturition.

Thanks to the Northern and Yorkshire $\mathrm{R}$ and $\mathrm{D}$ programme and Ferring Pharmaceuticals for financing the project, and to Dr M Penney and Dr Hampton of the Royal Gwent Hospital for performing the vasopressin assays.

1 Norgaard JP, Pedersen EB, Djurhuus JC. Diurnal antidiuretic hormone levels in enuretics. F Urol 1985;134:102931.

2 Rittig S, Knudsen UB, Norgaard JP, Pedersen EB, Djurhuus JC. Abnormal diurnal rhythm of plasma vasopressin and urinary output in patients with enuresis. Am f Physio 1989;256:F664-71.

3 A systematic review of the effectiveness of interventions for managing childhood nocturnal enuresis. NHS Centre for Reviews and Dissemination, University of York, 1997.

4 Moffatt ME, Harlos S, Kirshen AJ, Burd L. Desmopressin Moffatt ME, Harlos S, Kirshen AJ, Burd L. Desmopressin
acetate and nocturnal enuresis: how much do we know? acetate and nocturnal en
Pediatrics 1993;92:420-5.

5 Penney MD, Hampton D, Oleesky DA, Livingston C, Mulkerrin E. Radioimmunoassays for arginine vasopressin and atrial natiuretic peptide: application of a common protocol for plasma extraction using Sep-Pak $\mathrm{C}_{18}$ cartridges. Ann Clin Biochem 1992;29:652-8.

6 Swaab DF, Hofman MA, Honnebier MBOM. Development of vasopressin neurons in the human suprachiasmatic nucleus in relation to birth. Brain Res Dev Brain Res 1991; 52:289-93.

7 Wood CM, Butler RJ, Penney MD, Holland PC. Pulsatile release of arginine vasopressin (AVP) and it's effect on response to desmopressin in enuresis. Scand 7 Urol Nephrol Suppl 1994;163:93-101.

8 Makrides M, Neumann M, Simmer K, Pater J, Gibson R. Are long-chain polyunsaturated fatty acids essential Are long-chain polyunsaturated fatty acid

9 Kalo BB, Bella H. Enuresis: prevalence and associated facKalo BB, Bella $\mathrm{H}$. Enuresis: prevalence and associated fac-
tors among primary school children in Saudi Arabia. Acta Paediatr Scand 1996;85:1217-22.

10 Watanabe H. Sleep problems in children with nocturnal enuresis. Scand F Urol Nephrol Suppl 1995;173:55-8.

11 Ohne $\mathrm{T}$, et al. The increase in C-fos expression in vasopressin and oxytocin immunoreactive neurons in paraventricular and supraoptic nucleus of the hypothalamus following urinary retention. F Kyoto Pref Univ Med 1995;104:393-403.

12 Caione PL, Giorgio PL, Passerine-Glazel G, et al. Desmopressin (DDAVP) and oxybutynin in nocturnal enuresis: results of a multicentred trial. International Children's Continence Society monograph series No 1. Tunbridge Wells: Wells Medical Ltd, 1995.

13 Arnell H, Hjalmas K, Jagervall M, et al. The genetics of primary enuresis: inheritance and suggestion of second major mary enuresis: inheritance and suggestion of second major

14 Knepper MA. Molecular physiology or urinary concentrating mechanism: regulation of aquaporin water channels by vasopressin. Am $\mathcal{F}$ Physiol 1997;272:F3-12.

15 Butler RJ, Holland PC, Devitt H, Hiley E, Roberts G, Redfern E. The effectiveness of desmopressin in the treatment of childhood nocturnal enuresis: predicting response using pretreatment variables. BrF Urol 1998;81:29-36.

16 Jarvelin MR. Developmental history and neurological findngs in enuretic children. Dev Med Child Neurol 1989;31: 728-36.

17 Mathews SG, Lu F, Yang K, Challis JR. Hypothalamic pituitary adrenal function in the sheep fetus [review]. Reprod Fertil Dev 1995;7:509-16.

18 Jarvelin, et al. Etiological and precipitating factors for childhood enuresis. Acta Paediatr Scand 1991;80:361-9.

19 Ferguson DM, Horwood CT, Shanon FT. Secondary enuresis in a birth cohort of New Zealand children. Paed Perinat Epidemiol 1990;4:53-63. 